\title{
Multilocus sequence typing and ftsl sequencing: a powerful tool for surveillance of penicillin-binding protein 3-mediated beta-lactam resistance in nontypeable Haemophilus influenzae
}

Dagfinn Skaare ${ }^{1,2^{*}}$, Inger Lill Anthonisen ${ }^{1}$, Dominique A Caugant ${ }^{3,4}$, Andrew Jenkins ${ }^{5}$, Martin Steinbakk ${ }^{3}$, Linda Strand ${ }^{6}$, Arnfinn Sundsfjord ${ }^{2}$, Yngvar Tveten $^{6}$ and Bjørn-Erik Kristiansen ${ }^{2}$

\begin{abstract}
Background: Beta-lactam resistance in Haemophilus influenzae due to fts/ mutations causing altered penicillin-binding protein 3 (PBP3) is increasing worldwide. Low-level resistant isolates with the N526K substitution (group II low-rPBP3) predominate in most geographical regions, while high-level resistant isolates with the additional S385T substitution (group III high-rPBP3) are common in Japan and South Korea. Knowledge about the molecular epidemiology of rPBP3 strains is limited. We combined multilocus sequence typing (MLST) and fts//PBP3 typing to study the emergence and spread of rPBP3 in nontypeable H. influenzae (NTHi) in Norway.

Results: The prevalence of rPBP3 in a population of 795 eye, ear and respiratory isolates (99\% NTHi) from 2007 was $15 \%$. The prevalence of clinical PBP3-mediated resistance to ampicillin was $9 \%$, compared to $2.5 \%$ three years earlier. Group II low-rPBP3 predominated (96\%), with significant proportions of isolates non-susceptible to cefotaxime (6\%) and meropenem (20\%). Group III high-rPBP3 was identified for the first time in Northern Europe. Four MLST sequence types (ST) with characteristic, highly diverging fts/ alleles accounted for $61 \%$ of the rPBP3 isolates. The most prevalent substitution pattern (PBP3 type A) was present in $41 \%$ of rPBP3 isolates, mainly carried by ST367 and ST14. Several unrelated STs possessed identical copies of the fts/ allele encoding PBP3 type A. Infection sites, age groups, hospitalization rates and rPBP3 frequencies differed between STs and phylogenetic groups.

Conclusions: This study is the first to link fts/ alleles to STs in H. influenzae. The results indicate that horizontal gene transfer contributes to the emergence of rPBP3 by phylogeny restricted transformation.

Clonally related virulent rPBP3 strains are widely disseminated and high-level resistant isolates emerge in new geographical regions, threatening current empiric antibiotic treatment. The need of continuous monitoring of beta-lactam susceptibility and a global system for molecular surveillance of rPBP3 strains is underlined. Combining MLST and fts//PBP3 typing is a powerful tool for this purpose.
\end{abstract}

Keywords: Haemophilus influenzae, Beta-lactam resistance, ftsl, PBP3, BLNAR, MLST, PFGE, Horizontal gene transfer, Recombination, Surveillance

\footnotetext{
* Correspondence: dagfinn.skaare@siv.no

'Department of Microbiology, Vestfold Hospital Trust, Tønsberg, Norway

2University of Tromsø, Tromsø, Norway

Full list of author information is available at the end of the article
} 


\section{Background}

Haemophilus influenzae is a major cause of respiratory tract infections and invasive disease, with encapsulated strains of serotype b (Hib) being most virulent [1]. Nontypeable isolates (NTHi) now account for the majority of cases of invasive disease in countries where Hib conjugate vaccines have been introduced [2-4]. NTHi vaccines have a huge potential for further reducing the global burden of disease but are not yet available $[1,5]$.

Beta-lactams are first-line drugs for treatment of $H$. influenzae infections but resistance may develop due to transferable beta-lactamases (impacting penicillins only) or alterations in the transpeptidase domain of penicillinbinding protein 3 (PBP3), encoded by the ftsI gene (impacting all beta-lactams) [6]. Traditionally, isolates with the latter resistance mechanism have been denoted beta-lactamase negative ampicillin resistant (BLNAR), whereas isolates with both mechanisms have been denoted beta-lactamase positive amoxicillin-clavulanate resistant (BLPACR). PBP3-mediated resistance is defined by the presence of particular amino acid substitutions (Table 1): R517H or N526K near the KTG motif in low-level resistant isolates (groups I and II, respectively), and the additional substitution S385T near the SSN motif in highlevel resistant isolates (group III-like, S385T + R517H; group III, S385T + N526K) [7-10].

An increased prevalence of PBP3-mediated resistance (hereafter denoted rPBP3) has been observed worldwide [2,4,11-16]. Isolates with high-level resistance (high-rPBP3) are a major clinical problem in Japan and South Korea [15-17] whereas low-level resistant (low-rPBP3) isolates so far predominate in the rest of the world [4,11,12,14,18-21].

Table 1 Genotypes of PBP3-mediated resistance in Haemophilus influenzae

\begin{tabular}{|c|c|c|c|c|c|}
\hline \multirow{2}{*}{\multicolumn{3}{|c|}{ Genotype designations $^{a}$}} & \multicolumn{3}{|c|}{ PBP3 substitutions ${ }^{b}$} \\
\hline & & & \multirow{2}{*}{$\frac{\text { SSN }}{\text { S385 }}$} & \multicolumn{2}{|c|}{ KTG } \\
\hline Category $^{c}$ & Level & Group & & R517 & N526 \\
\hline \multirow[t]{4}{*}{ rPBP3 } & High & \|\|$^{d}$ & $\mathrm{~T}$ & & K \\
\hline & & III-like $e^{e}$ & $\mathrm{~T}$ & $\mathrm{H}$ & \\
\hline & Low & $\|$ & & & K \\
\hline & & । & & $\mathrm{H}$ & \\
\hline
\end{tabular}

sPBP3 NA NA

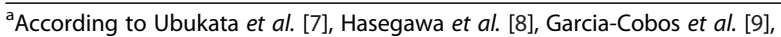
Hotomi et al. [10] and this study. NA, not applicable.

${ }^{b}$ Essential amino acid substitutions in PBP3 (transpeptidase domain, 338-573) with the amino acid sequence of $H$. influenzae Rd KW20 [GenBank:U32793] as reference. SSN, Ser-Ser-Asn motif; KTG, Lys-Thr-Gly motif.

crPBP3, isolates with PBP3 sequences conferring resistance to beta-lactams (isolates assigned to groups I, II, III-like and III); SPBP3, isolates with PBP3 sequences conferring wild-type susceptibility to beta-lactams (remaining isolates).

${ }^{\mathrm{d}}$ Originally reserved for isolates with the additional substitutions M377I and L389F by Ubukata et al. [7], modification proposed by Hotomi et al. [10]. eOriginally categorized as group I by Ubukata et al. [7], new group assignment proposed by Garcia-Cobos et al. [9].
Group II isolates with a characteristic substitution pattern, PBP3 type A (D350N, M377I, A502V, N526K, V547I and N569S) [11], and compatible patterns (identical to PBP3 type A as far as comparison is possible) are particularly common $[3,4,9,11,12,16,18,20,22-25]$. The mechanisms by which rPBP3 isolates emerge are not fully understood. Spontaneous mutations are considered the primary cause of the substitutions R517H, N526K and S385T [6,26] but horizontal gene transfer (HGT) by classical transformation and homologous recombination has been suggested to play an important role in the further development and spread of resistance [11,26-28].

Clonal spread of rPBP3-NTHi is extensively documented [3,4,6,9-11,18,26]. However, knowledge about the molecular epidemiology of rPBP3 strains is limited. Previous studies based on pulsed-field gel electrophoresis (PFGE) and other molecular methods have generated results not easily compared between studies. Multilocus sequence typing (MLST) has the advantage of providing objective, unambiguous data, easy to compare and well suited for assessment of phylogenetic relationship in both encapsulated isolates and NTHi [29,30]. The MLST scheme for $H$. influenzae assigns isolates to sequence types (ST) based on allelic profiles of the seven housekeeping genes $a d k, \operatorname{atp} G, \operatorname{frdB}$, fucK, $m d h, p g i$ and $\operatorname{rec} A$ [30]. Software for phylogenetic analysis and a continuously updated database with STs, serotypes and clinical data (but not resistance genotypes) is available on the website http://haemophilus.mlst.net. MLST has improved our understanding of population structure in $H$. influenzae [29-32]. A maximum-parsimony analysis of concatenated sequences from all isolates in the database has identified 14 phylogenetic groups (Clades 1-13 and eBURST group 2) with different genetic characteristics, including serotypes and virulence determinants [32].

The objectives of this study were to: 1) Estimate the prevalence of rPBP3 in eye, ear and respiratory isolates of $H$. influenzae in Norway and map PBP3 genotypes and phenotypic beta-lactam susceptibility profiles; 2) Examine the molecular epidemiology of rPBP3 isolates and seek for evidence of HGT; and 3) Explore any associations between phylogeny, resistance genotypes and pathogenicity, as reflected by clinical characteristics (age, gender, hospitalization rates and sample types).

\section{Methods}

\section{Bacterial isolates}

One hundred and seventy-seven $H$. influenzae isolates with a phenotype suggesting rPBP3 (Resistant group, R-group) and 19 isolates with wild-type susceptibility to beta-lactams (Susceptible group, S-group) were characterized. The isolates were selected from a population of 808 consecutive eye, ear and respiratory tract isolates, collected as part of standard patient care in January and 
February 2007, included in the Norwegian Surveillance Programme for Antimicrobial Resistance (NORM) [33]. Selection was based on the susceptibility profiles reported by the primary laboratories (Figure 1). Thirteen isolates were selected but excluded for various reasons. Clinical information (site of isolation; age and gender of the patient; hospitalization status at the time of sampling) for the 196 study isolates and 599 isolates in the original population was used for statistical analyses.

PFGE band patterns and ftsI sequences for $46 \mathrm{H}$. influenzae isolates from a comparable population collected in 2004, characterized in a previous study [11], were included in the phylogenetic analyses.

\section{Species identification and serotyping}

Isolates were inoculated on chocolate agar and incubated overnight at $35 \pm 1^{\circ} \mathrm{C}$ in ambient air with $5 \% \mathrm{CO}_{2}$. After control of purity and presumptive identification by smell, colony morphology and dependence of $\beta$-NAD and haemin, isolates were frozen at $-70^{\circ} \mathrm{C}$ using Microbank vials (Pro-Lab Diagnostics, Richmond Hill, Ontario, Canada).
Species identification was confirmed by outer membrane protein P6 (ompP6) and 16S rRNA PCR using primers as described previously [34] and probes designed for this study (Table 2). Where this test was negative $(n=10)$, a $547 \mathrm{bp}$ fragment of the 16S rRNA gene was sequenced at GATC Biotech (Konstanz, Germany) to confirm species identification.

Capsular serotyping was done by bexA PCR and capsule type-specific PCRs for bexA positive isolates as described previously [35], with modifications to the HI-1, HI-2 and $\mathrm{f} 3$ primers. A new serotype e-specific reverse primer and a bexA probe were designed for this study (Table 2).

\section{Susceptibility testing}

MIC determination by microbroth dilution (HTM, Oxoid Ltd, Basingstoke, UK) was carried out according to CLSI guidelines [36], except that testing of penicillinbeta-lactamase inhibitor combinations was performed with fixed inhibitor concentrations [37]. Beta-lactam agents tested were ampicillin, amoxicillin, piperacillin,

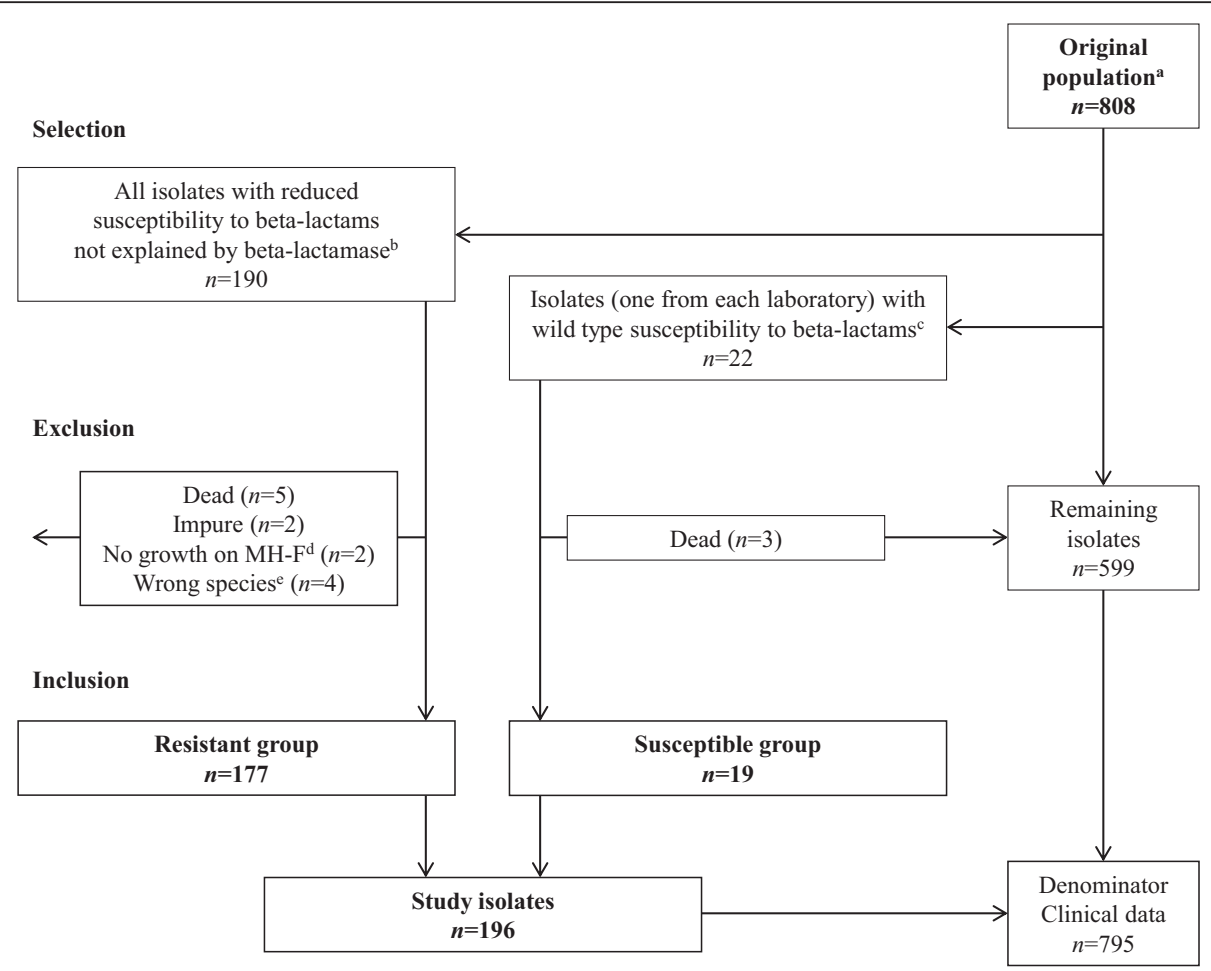

Figure 1 Study isolates. Flowchart showing selection and inclusion of bacterial isolates. ${ }^{a}$ NORM 2007 surveillance population [33]. ${ }^{b}$ According to phenotypic susceptibility profiles (by gradient MIC, disk diffusion and beta-lactamase detection) as reported by the primary laboratories. The following selection criteria were used: amoxicillin-clavulanate MIC $\geq 2 \mathrm{mg} / \mathrm{L}$, cefuroxime MIC $\geq 4 \mathrm{mg} / \mathrm{L}$, cefotaxime MIC $\geq 0.12 \mathrm{mg} / \mathrm{L}$ and/or cefaclor $30 \mu \mathrm{g}$ zone $<17 \mathrm{~mm}$ (all isolates); and ampicillin MIC $\geq 1 \mathrm{mg} / \mathrm{L}$, phenoxymethylpenicillin $10 \mu \mathrm{g}$ zone $<13 \mathrm{~mm}$ and/or ampicillin $2 \mu \mathrm{g}$ zone $<16 \mathrm{~mm}$ (beta-lactamase negative isolates). The selection criteria were constructed using epidemiological cut-off MIC values defined by EUCAST (www.eucast.org/MIC_distributions) and zone diameter distributions from the surveillance report [33]. Information about the methodologies for susceptibility testing are included in the surveillance report [33]. ' One beta-lactamase negative isolate from each laboratory, randomly selected from the isolates remaining after selection for the Resistant group. ${ }^{\mathrm{d}} \mathrm{MH}-\mathrm{F}$, Mueller-Hinton agar supplemented with defibrinated horse blood and $\beta$-NAD for susceptibility testing of fastidious organisms (www.eucast.org). ${ }^{e}$. parainfluenzae $(n=3)$ and H. haemolyticus $(n=1)$. 
Table 2 New and modified primers and probes used in this study

\begin{tabular}{|c|c|c|c|c|}
\hline Name & Function & Target & Sequences $\left(5^{\prime} \text { to } 3^{\prime}\right)^{a}$ & Original (reference) \\
\hline bexAFb & F-primer & bexA & CGTTTATRTGATGTTGATCCTGA & $\mathrm{HI}-1[35]$ \\
\hline bexARb & R-primer & bexA & TGTCCATATCTTCAAAATGGTG & $\mathrm{HI}-2[35]$ \\
\hline bexAP & Probe & bexA & FAM ATGCAAGYCGRGCTITCATCCCTG-BHQ & This study \\
\hline Hinf_fR & R-primer & cap (serotype f) & GGTACTATCAAGTCCAAATC & f3 [35] \\
\hline Hinf_eR2 & R-primer & cap (serotype e) & CTAATTGTTCTITCTGTCTA & This study \\
\hline ompP6P & Probe & ompP6 & ACG TGG TAC ACC AGA ATA CAA CAT CGA & This study \\
\hline H16SP & Probe & 165 rRNA gene & TCGCTCCACCTCGCAGCTTCGCT & This study \\
\hline TEMP & Probe & $b l a_{\mathrm{TEM}}$ & CAG CTC CGG TTC CCA ACG ATC AAG & This study \\
\hline ROBP & Probe & $b l a_{\mathrm{ROB}}$ & TAG CGA CAA CAG CGC GAC CAA TाT G & This study \\
\hline
\end{tabular}

${ }^{\mathrm{a}}$ Sites of modifications in bold.

cefuroxime, cefotaxime (Sigma-Aldrich, St. Louis, MO, USA) and meropenem (Sequoia, Pangbourne, UK). For beta-lactamase positive isolates, ampicillin, amoxicillin and piperacillin MICs were determined in the presence of sulbactam $4 \mathrm{mg} / \mathrm{L}$ (Sequoia), clavulanate $2 \mathrm{mg} / \mathrm{L}$ and tazobactam $4 \mathrm{mg} / \mathrm{L}$ (Sigma-Aldrich), respectively. MICs were within accepted ranges for $H$. influenzae ATCC 49247 (rPBP3) and H. influenzae ATCC 49766 (sPBP3), and within the wild type range (www.eucast.org/MIC_distributions) for $H$. influenzae ATCC 35056 (TEM-1 positive).

MICs were interpreted according to EUCAST clinical breakpoints, except for piperacillin and piperacillin-tazobactam where breakpoints are not defined [37]. Meningitis breakpoints were used for susceptibility categorization of meropenem to allow quantification of low-level resistance. Data from this study are included in the EUCAST database for MIC distributions of clinical isolates.

\section{Resistance genotyping}

PCR and sequencing of the transpeptidase domain of the ftsI gene were performed as described previously [11]. DNA sequences were analysed using Lasergene software (DNASTAR, Madison, WI, USA) and the sequences (nucleotides 1010-1719) have been deposited in the EMBL Nucleotide Sequence Database [EMBL:HG818627818822].

An UPGMA (unweighted pair group method with arithmetic mean) phylogram of ftsI alleles from this and a previous study [11] was constructed by distance methods using ClustalW2 (www.ebi.ac.uk) and displayed using TreeDyn software (www.phylogeny.fr) with $H$. parainfluenzae [EMBL:AB267856] as outgroup (Figure 2). Clusters of closely related alleles were assigned Greek letters (alpha - pi) with numbers denominating alleles within each cluster.

According to PBP3 substitution patterns (Table 1), isolates were categorized into resistance genotypes (Table 3). Group II rPBP3 isolates and isolates lacking essential substitutions (denoted sPBP3) were assigned to PBP3 types
(A - Q and z1 - z13, respectively) according to the previously established system [11], further developed in this study.

The DNA and PBP3 sequences of $H$. influenzae $\mathrm{Rd}$ KW20 [GenBank:U32793] were used as references (alpha-0 and $\mathrm{z} 0$, respectively).

Isolates reported as beta-lactamase positive by the primary laboratory and isolates with a phenotype suggesting beta-lactamase production were examined by TEM-1 and ROB-1 PCR as described previously [38], with detection of PCR products by probes designed for this study (Table 2).

\section{Molecular strain characterization}

MLST was performed by standard procedures with sequencing of internal fragments of the seven housekeeping genes $a d k, a t p G, f r d B, f u c K, m d h, p g i$ and $r e c A$ [30]. Following registration of sequences at http://hae mophilus.mlst.net for assignment of allele numbers and STs, data were analysed using software available on the website, with the construction of an UPGMA dendrogram based on the pairwise differences in allelic profiles (Figure 3), and division of STs into clonal complexes (CC) using eBURSTv3. The criterion for assignment to a $\mathrm{CC}$ (named according to the predicted founder) was sequence identity with another member of the complex at at least six loci [31].

STs were assigned to phylogenetic groups (here denoted phylogroups) according to previously performed maximum parsimony analysis of all STs in the MLST database [32]. More recent STs, not encompassed by the parsimony analysis, were indirectly assigned to phylogroups if they belonged to CCs encompassing STs with known phylogroup.

PFGE of the 177 isolates in the R-group was carried out as described previously [11,38]. A dendrogram of band patterns, with 46 isolates from our previous study included [11], was constructed using GelCompare II software (Applied Maths, Sint-Martens-Latem, Belgium), 


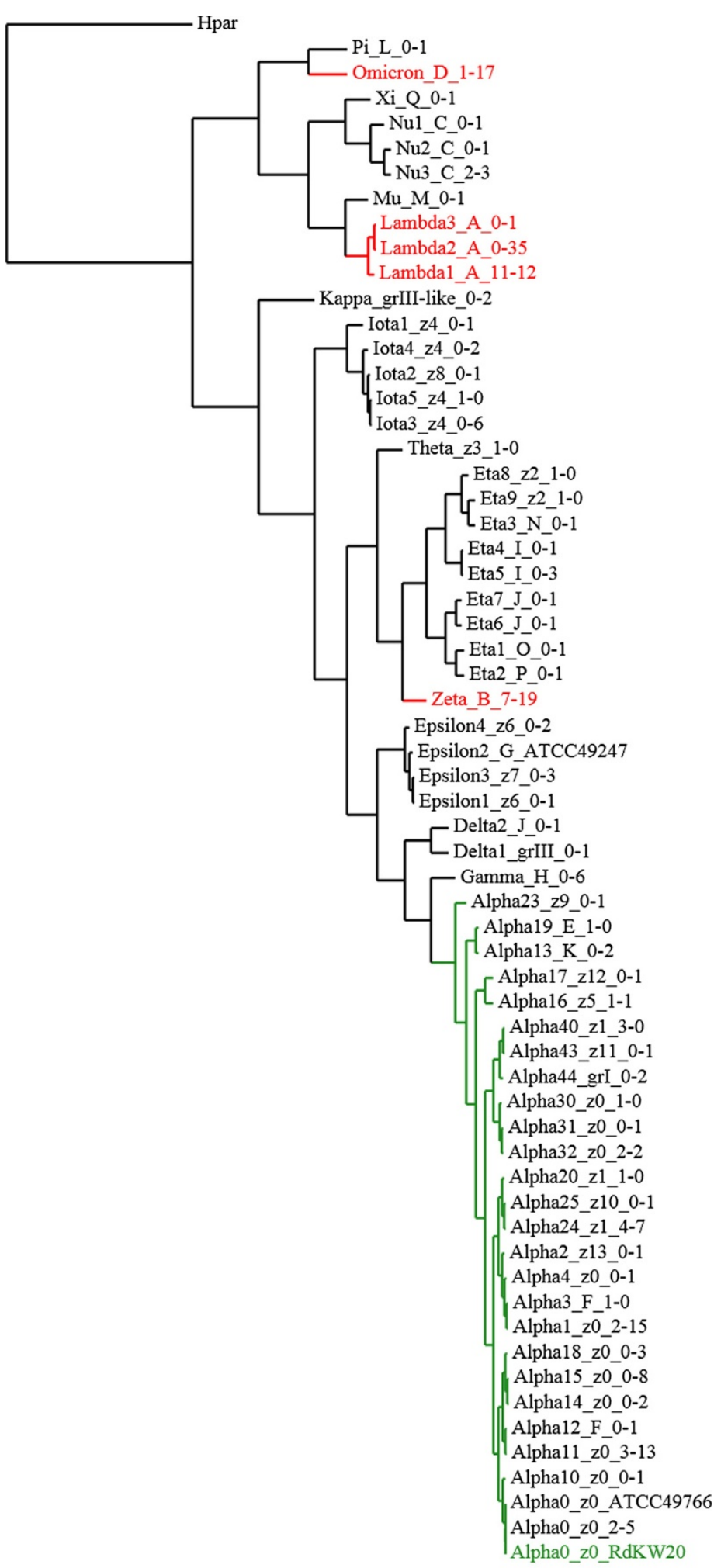

Figure 2 (See legend on next page.) 
(See figure on previous page.)

Figure 2 ftsl phylogram. UPGMA phylogram of fts/ DNA sequences (transpeptidase domain, nucleotides 1010-1719) in the current $(n=196)$ and previous study $(n=46)$ [11]. The outgroup (Hpar) is $H$. parainfluenzae [EMBL:AB267856] and the reference sequence $(z 0)$ is $H$. influenzae Rd KW20 [GenBank:U32793]. The H. influenzae reference strains ATCC 49247 and ATCC 49766 are also included. The scale is DNA sequence divergence ( $0.05=5 \%$ divergence). Labels indicate fts/ alleles, PBP3 types and number of isolates with the particular allele in the previous and current study, respectively. The reference cluster alpha (green) and the alleles encoding PBP3 types A, B and D (red) are highlighted.

Dice coefficients of similarity and the UPGMA algorithm (Figure 4). Clusters of related or possibly related isolates were identified by comparison of band patterns [39] and numbered according to the system established previously [11].

\section{Statistics}

Multivariate regression analysis and Fisher's exact test was performed using Predictive Analytics Software (PASW) Statistics version 17.0 (IBM Corporation, US).

\section{Ethics}

The bacterial isolates and patient information used in this study were collected as part of the Norwegian Surveillance Programme for Antimicrobial Resistance (NORM). The NORM programme is warranted in Norwegian law (http://lovdata.no, FOR-2003-11-14-1353) and no further ethical approval was required for the use of isolates and data in this study.

\section{Results}

\section{Resistance genotypes}

In the R-group ( $\mathrm{n}=177), 116$ isolates $(66 \%)$ had essential PBP3 substitutions and were categorized as rPBP3. The remaining 61 isolates in the R-group, and all 19 isolates in the S-group, lacked essential substitutions and were categorized as SPBP3 (Table 4).

Most rPBP3 isolates were group II (111/116, 96\%), including seven TEM-1 positive isolates, but one group III and two group III-like high-rPBP3 isolates were also identified (Table 3). The rPBP3 prevalence in the original population was thus $15 \%(116 / 795)$ and the prevalence of combined rPBP3 and TEM-1 was 0.9\% (7/795).

Eighteen PBP3 substitution patterns were present in rPBP3 isolates, with PBP3 types A, B and D accounting for $72 \%(84 / 116)$ and PBP3 type A alone accounting for $41 \%$ (48/116). The N526K substitution was encoded by the DNA triplets AAA and AAG in 54\% (61/112) and $46 \%(51 / 112)$ of the cases, respectively.

Analysis of the $49 \mathrm{ftsI}$ alleles in the current study identified 14 clusters (Figure 2). PBP3 types A, B and D were confined to distinct clusters (lambda, zeta and omicron), all highly divergent from the reference sequence. Type A was encoded by three closely related alleles (cluster lambda) whereas types B (zeta) and D (omicron) showed no allelic diversity. Several clusters encompassed more than one PBP3 type, but only type J appeared in more than one cluster (eta and delta). The lambda-1 and zeta alleles, encoding PBP3 types A and B, respectively, were highly prevalent in both sampling periods.

\section{Serotypes and phylogeny}

Except for two serotype $\mathrm{f}$ (Hif) ear and respiratory tract isolates, all study isolates were nontypeable.

The 196 isolates represented 70 STs; hereunder 15 novel (ST1190 through ST1204, represented by one isolate each) (Figure 3). Eight STs had $>5$ representatives and accounted for $54 \%(105 / 196)$ of the isolates (Table 5). By eBURST analysis, the STs were grouped into 39 clonal complexes (CC) and three singletons.

Direct assessment of phylogroup was possible for 32 STs (accounting for 129 isolates) and indirect assignment was possible for 30 STs (55 isolates). Eight STs (12 isolates) could not be assigned to a phylogroup. Ten out of 14 recognized phylogroups [32] were represented, and $69 \%$ of the isolates belonged to Clade $13(n=59)$, eBURST group $2(n=50)$ and Clade $9(n=26)$. The two Hif isolates (sPBP3, ST124) were in Clade 2.

The S-group was more diverse than the R-group and differed phylogenetically: fifteen STs were represented among 19 S-group isolates, with only one, ST159, being among the eight most frequent STs overall (Table 5). Two major R-group phylogroups (eBURST group 2 and Clade 8) were absent from the S-group.

Eight PFGE clusters of $>5$ isolates were identified, with Dice coefficients of clustering between $71 \%$ and $76 \%$ (Figure 4). PFGE clusters corresponded well to CCs, occasionally with a higher or lower resolution level. Additionally, two clusters (6B and 12) suggested genetic relationship (by three band difference) between isolates assigned to phylogroups (eBURST group 2 and Clade 13, respectively) and isolates with no phylogroup assignment, probably reflecting distant phylogenetic relationship not detected by the parsimony analysis.

\section{Phylogeny and resistance genotypes}

The $116 \mathrm{rPBP} 3$ and 80 sPBP3 isolates were distributed on 32 and 44 STs, respectively. Six of the 70 STs in this study (ST12, ST57, ST155, ST159, ST411 and ST422) included both categories. Most rPBP3 isolates (102/116, $88 \%$ ) belonged to five phylogroups (rPBP3 proportions in brackets): eBURST group 2 (45/50, 90\%); Clade 13 (28/59, 47\%); Clade 9 (22/26, 85\%); Clade 8 (5/8, 63\%) or Clade $10(2 / 4,50 \%)$. The remaining $14 \mathrm{rPBP} 3$ isolates 
Table 3 Resistance genotypes, PBP3 types and PBP3 substitutions

\begin{tabular}{|c|c|c|c|c|c|c|c|c|c|c|c|c|c|c|c|c|c|c|c|c|c|c|c|}
\hline \multirow{3}{*}{$\begin{array}{l}\text { Resistance } \\
\text { genotypes }^{\mathrm{a}}\end{array}$} & \multirow{3}{*}{$\begin{array}{c}\text { PBP3 } \\
\text { types }^{b}\end{array}$} & \multirow[t]{3}{*}{$n^{c}$} & \multirow[t]{3}{*}{$\mathrm{Sg}^{\mathrm{d}}$} & \multirow[t]{3}{*}{$\mathrm{Bla}^{\mathrm{e}}$} & \multicolumn{19}{|c|}{ PBP3 substitutions ${ }^{f}$} \\
\hline & & & & & D & $S$ & $A$ & $M$ & $S$ & $P$ & $A$ & 1 & G & A & v & $\mathbf{R}$ & $N$ & A & $T$ & V & D & $A$ & $N$ \\
\hline & & & & & 350 & 357 & 368 & 377 & 385 & 392 & 437 & 449 & 490 & 502 & 511 & 517 & 526 & 530 & 532 & 547 & 551 & 554 & 569 \\
\hline \multicolumn{24}{|l|}{ High-rPBP3 } \\
\hline Group III & - & 1 & & & $N$ & N & & & $T$ & & & & & $\mathrm{~T}$ & & & $\mathbf{K}^{g}$ & & & । & & & S \\
\hline Group III-like & - & 2 & & & $N$ & N & & 1 & $\mathbf{T}$ & & & & & & & $\mathrm{H}$ & & & $S$ & I & & & \\
\hline \multicolumn{24}{|l|}{ Low-rPBP3 } \\
\hline \multirow[t]{15}{*}{ Group II } & A & 48 & & 1 & N & & & I & & & & & & v & & & $\mathbf{K}^{h}$ & & & I & & & S \\
\hline & B & 19 & & 5 & & & & & & & & V & & & & & $\mathbf{K}^{\mathrm{g}}$ & & & I & & & S \\
\hline & C & 5 & & & N & & & । & & & & & $E$ & & & & $\mathbf{K}^{\mathrm{h}}$ & & & । & & & S \\
\hline & $\mathrm{D}$ & 17 & & & $N$ & & & & & & & & $\mathrm{E}$ & & & & $\mathbf{K}^{9}$ & S & & & & & \\
\hline & $\mathrm{F}$ & 1 & & & & & & & & & & & & & & & $\mathbf{K}^{9}$ & & & & & & \\
\hline & $\mathrm{H}$ & 6 & & & & & & & & & & & & v & & & $\mathbf{K}^{h}$ & & & & & & \\
\hline & I & 4 & & & $N$ & & & & & & $S$ & & & v & & & $\mathbf{K}^{\mathrm{g}}$ & & & I & & & S \\
\hline & J & 3 & & & $N$ & & & & & & & & & $\mathrm{~T}$ & & & $\mathbf{K}^{\mathrm{g}}$ & & & I & & & $\mathrm{S}$ \\
\hline & K & 2 & & & & & $\mathrm{~T}$ & & & & & & & $\mathrm{~T}$ & & & $\mathbf{K}^{9}$ & & & & & & \\
\hline & L & 1 & & & $N$ & & & & & & & & $E$ & & & & $\mathbf{K}^{\mathrm{g}}$ & & & I & & $D^{i}$ & S \\
\hline & M & 1 & & & $N$ & & & & & & & & & v & & & $\mathbf{K}^{\mathrm{h}}$ & & & I & & & $\mathrm{S}$ \\
\hline & $N$ & 1 & & 1 & $\mathrm{~N}$ & & & & & & $S$ & V & & & & & $\mathbf{K}^{9}$ & & & I & & & $\mathrm{S}$ \\
\hline & $\mathrm{O}$ & 1 & & & & & & & & & & & & $\mathrm{~T}$ & & & $\mathbf{K}^{\mathrm{g}}$ & & & I & & & $\mathrm{S}$ \\
\hline & P & 1 & & & & & & & & & & & & $\mathrm{~T}$ & & & $\mathbf{K}^{\mathrm{g}}$ & & & I & & & \\
\hline & Q & 1 & & & & & & & & & & & E & v & & & $\mathbf{K}^{\mathrm{h}}$ & & & I & & & S \\
\hline Group I & - & 2 & & & & & & & & & & & & & & $\mathrm{H}$ & & & & I & & $\mathrm{T}$ & \\
\hline \multirow[t]{12}{*}{ sPBP3 } & z0 & 51 & 15 & 6 & & & & & & & & & & & & & & & & & & & \\
\hline & z4 & 9 & 1 & & $\mathrm{~N}$ & & & & & & & & & & & & & & & I & & & S \\
\hline & z1 & 7 & 3 & 2 & & & & & & & & & & & & & & & & I & & & \\
\hline & $z 6$ & 3 & & & & & & & & & & & & & & & & & & I & & & S \\
\hline & z7 & 3 & & & & & & & & & & & & & & & & & & I & & $\mathrm{T}$ & S \\
\hline & z5 & 1 & & 1 & & & & & & & & & & $S$ & & & & & & & & & \\
\hline & z8 & 1 & & & $N$ & & & & & & & & & $\mathrm{~T}$ & & & & & & 1 & & & S \\
\hline & z9 & 1 & & & $N$ & & & & & & & & & & & & & & & I & & & \\
\hline & $z 10$ & 1 & & & & & & & & & & & & & & & & & & I & $A^{i}$ & & \\
\hline & z11 & 1 & & & & & & & & & & & & & A & & & & & I & & & \\
\hline & $z 12$ & 1 & & & & & & & & $S^{i}$ & & & & & & & & & & & & & \\
\hline & $z 13$ & 1 & & & & & & & & & & & & $\mathrm{~T}$ & & & & & & & & & \\
\hline
\end{tabular}

${ }^{\mathrm{a}}$ See Table 1.

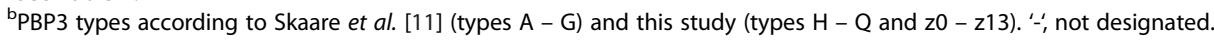

c $n$, No. of study isolates.

${ }^{\mathrm{d}} \mathrm{Sg}$, No. of isolates from the Susceptible group included in $n$.

e Bla, No. of beta-lactamase positive isolates (all TEM-1) included in $n$.

${ }^{\mathrm{f}}$ Amino acid substitutions in PBP3 (transpeptidase domain, 338-573) with the amino acid sequence of $H$. influenzae Rd KW20 [GenBank:U32793] as reference (z0).

Essential substitutions in bold.

${ }^{9} \mathrm{~N} 526 \mathrm{~K}$ encoded by the DNA triplet AAG.

${ }^{\mathrm{h}} \mathrm{N} 526 \mathrm{~K}$ encoded by the DNA triplet AAA.

iNovel substitution.

lacked phylogroup assignment. The two group III-like and the single group III high-rPBP3 isolates were ST160 (no phylogroup) and ST1197 (Clade 13), respectively. No isolates in Clade $1(n=5)$, Clade $2(n=4)$, Clade 6 $(n=1)$, Clade $11 \quad(n=5)$ and Clade $12(n=2)$ were rPBP3.

The ftsI alleles lambda-2, zeta and omicron, encoding the three most frequent PBP3 types A, B and D, 


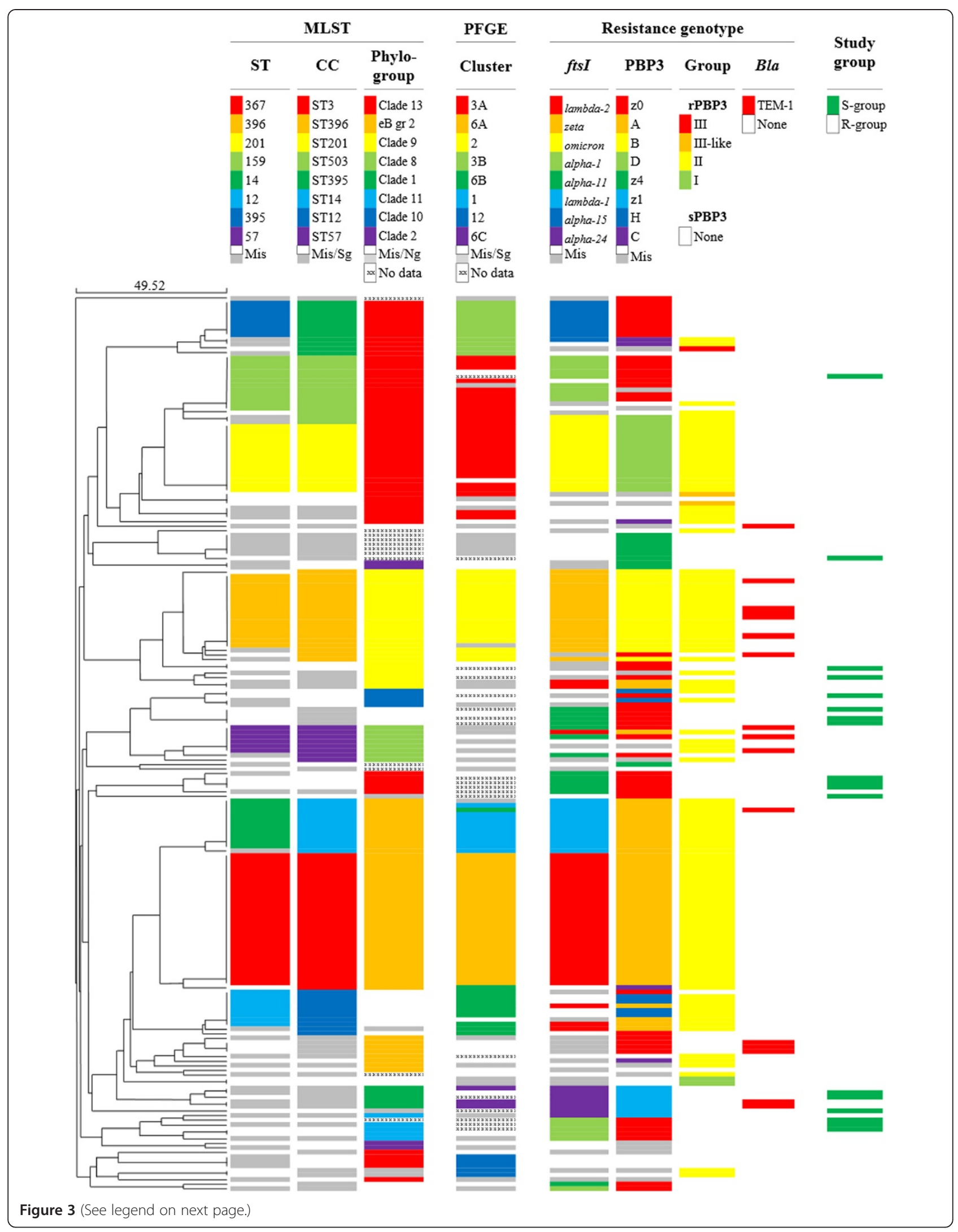


(See figure on previous page.)

Figure 3 MLST dendrogram. The correlation between phylogenetic groups (MLST and PFGE) and resistance genotypes. UPGMA dendrogram of STs based on pair-wise differences in allelic profiles of the 196 study isolates with additional information about CCs, phylogroups, PFGE clusters, $\mathrm{fts}$ alleles, PBP3 types, PBP3 groups, beta-lactamase and study groups. The colour scale indicates relative frequencies of various alternatives within each of the columns 1-6. eB gr2, eBURST group 2; Mis, miscellaneous; Sg, singletons; Ng, no phylogroup; S-group, Susceptible group; R-group, Resistant group.

respectively, were, with a few notable exceptions, carried by ST367 (eBURST group 2), ST396 (Clade 9) and ST201 (Clade 13) (Figure 3). In addition, PBP3 type A encoded by the slightly different allele lambda-1 was present in ST14, a triple locus variant of ST367 (both STs belong to eBURST group 2). These four strains (defined by combinations of STs and ftsI alleles) accounted for 61\% (71/116) of the rPBP3 isolates in the current study.

Two strains frequently occurring in this study (ST14 with PBP3 type A and ST396 with PBP3 type B) had PFGE band patterns and $f t s I$ alleles identical to strains in the two most prevalent resistant clones three years earlier (PFGE clusters 1 and 2, respectively) (Figure 4) [11].

Apart from ST367, PBP3 type A encoded by lambda-2 was present in the following unrelated STs: ST57 (Clade 8), ST85 (Clade 9) and ST12 (no phylogroup). Similarly, the ftsI allele gamma, encoding PBP3 type $\mathrm{H}$, was present in ST12 (no phylogroup) as well as the unrelated ST411 and ST422 (Clade 10). Conversely, seven STs hosted more than one PBP3 type. Notably, the six ST57 isolates carried four highly divergent $\mathrm{rPBP} 3$ types $(\mathrm{A}, \mathrm{K}, \mathrm{L}$ and $\mathrm{N})$ and the reference sequence $(\mathrm{z} 0)$.

Three ST57 isolates were TEM-1 positive but only one isolate had both TEM-1 and rPBP3. Most isolates with both resistance mechanisms (5/7, 71\%) were ST396.

\section{Clinical characteristics}

Clinical information for the 196 study isolates and the 599 remaining isolates in the original population is summarized in Table 4. For the study isolates, median age and age range of the patients were $5(0-86)$ yrs with a male/female ratio of 1.0. The corresponding numbers in the original population were $5(0-97)$ and 1.0.

Multivariate regression analysis of isolates with known hospitalization status $(766 / 795,96 \%)$ showed that increasing age $(\mathrm{OR}=1.3, \mathrm{p}<0.001)$ and male gender $(\mathrm{OR}=1.8, \mathrm{p}=0.001)$ were significant independent risk factors for hospitalization. With adjustment for age, gender and beta-lactamase production, there was a borderline significant association between rPBP3 and hospitalization $(\mathrm{OR}=1.6, \mathrm{p}=0.053)$. Similarly, multivariate analysis of isolates with known site of isolation (768/795, 97\%) showed a significant association between rPBP3 and eye infection (OR $=2.1, \mathrm{p}=0.003)$ but no association with other localizations. Information about STs was available for study isolates only and thus not included in the regression analysis.
The eight most prevalent STs were highly diverse with respect to resistance genotypes and clinical characteristics (Table 5). There was no correlation between rPBP3 proportions and hospitalization rates in the various STs. Three STs, two of which consisting entirely of rPBP3 isolates (ST396 and ST201) were significantly associated with eye infection $(\mathrm{p}<0.05)$. ST396 was also significantly associated with the age group $0-3$ yrs $(\mathrm{p}=0.004)$.

\section{Beta-lactam susceptibility}

Median MICs $\left(\mathrm{MIC}_{50}\right)$ were generally $\geq 2$ dilution steps higher in group II rPBP3 isolates than in sPBP3 isolates (Table 6). The single group III high-rPBP3 isolate had MICs $\geq 2$ steps higher than $\mathrm{MIC}_{50}$ in group II isolates. $\mathrm{MIC}_{50}$ for cefotaxime differed slightly between isolates with PBP3 types A $(0.03 \mathrm{mg} / \mathrm{L}), B(0.016 \mathrm{mg} / \mathrm{L})$ and D $(0.06 \mathrm{mg} / \mathrm{L})$. There were otherwise no significant differences (within \pm 1 dilution step) between $\mathrm{MIC}_{50}$ in various PBP3 types, nor between sPBP3 isolates in the two study groups.

The majority of group II isolates had MICs above the S-breakpoints for ampicillin, amoxicillin and cefuroxime. Significant proportions were resistant to cefotaxime $(7 / 111,6 \%)$ and non-susceptible to meropenem (22/111, $20 \%$ ), with representatives from all four major rPBP3 strains. Notably, 12\% (13/111) of group II isolates were categorized as susceptible to all agents, whereas $24 \%$ $(19 / 80)$ of sPBP3 isolates were non-susceptible to $\geq 1$ beta-lactam, most commonly intermediately susceptible to cefuroxime $(n=10)$. No association with ST or phylogroup was observed.

The prevalences of clinical PBP3-mediated resistance to ampicillin and cefotaxime and non-susceptibility to meropenem in the original population $(n=795)$ were $9 \%, 1.3 \%$ and $2.9 \%$, respectively.

\section{Discussion}

\section{Resistance epidemiology}

We found a $15 \%$ prevalence of $\mathrm{rPBP} 3$ in a nationwide collection of 795 eye, ear and respiratory isolates of $H$. influenzae in Norway. The prevalence of clinical resistance to ampicillin due to rPBP3 was 9\%, compared to $2.5 \%$ in a similar study three years earlier [11]. Despite methodological differences between the two studies, we conclude with a significant increase from 2004 to 2007. National phenotypic surveillance data indicate a further increase to $17 \%$ rPBP3 in respiratory isolates in 2011 [40] 


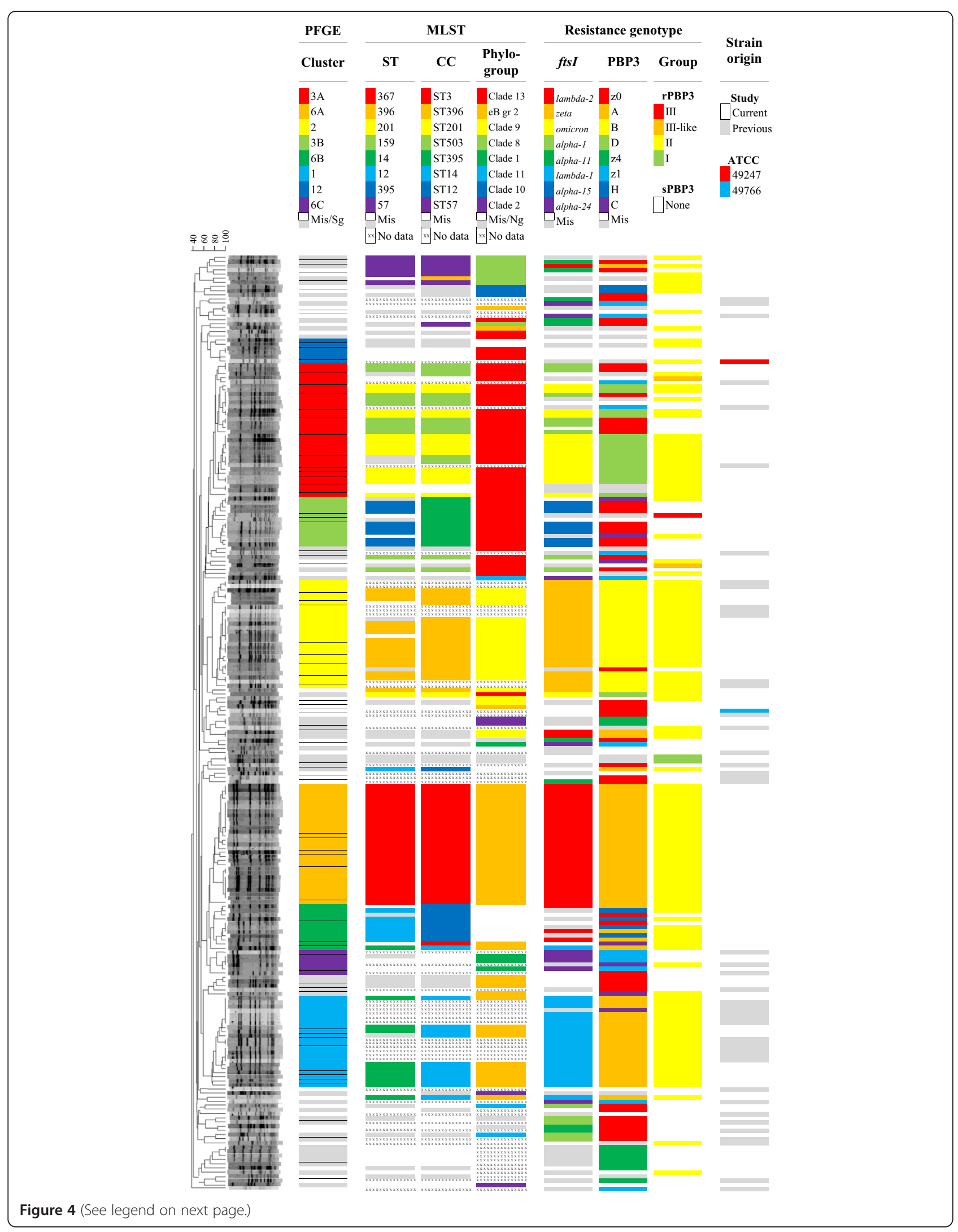


(See figure on previous page.)

Figure 4 PFGE dendrogram. The correlation between phylogenetic groups (PFGE and MLST) and resistance genotypes. UPGMA dendrogram of band patterns for the 177 isolates in the Resistant group and 46 isolates from a previous study [11]. Clusters of related or possibly related isolates by analysis of band patterns and Dice coefficient of similarity are indicated by colours. Horizontal lines separate different band patterns. Additional information about STs, CCs, phylogroups, ftsl alleles, PBP3 types, PBP3 groups and strain origin is provided. The colour scale (similar to Figure 3) indicates relative frequencies of various alternatives within each of the columns 1-6. eB gr2, eBURST group 2; Mis, miscellaneous; Sg, singletons; $\mathrm{Ng}$, no phylogroup.

and a prevalence at 15\% rPBP3 in invasive isolates in 2012 ( $n=73,77 \%$ nontypeable) [41], consistent with observations in other European countries and in Canada $[2,4,12,14]$.

As expected, group II low-level resistant isolates predominated. Notably, group III high-rPBP3 was identified for the first time in Northern Europe. The genotypic distinction between low-level and high-level beta-lactam resistance is clinically relevant: As resistance to cefotaxime is mainly seen in high-rPBP3 [6], cefotaxime is suitable for empiric treatment of severe disease only in regions where high-rPBP3 is rare. However, $6 \%$ of group II isolates in the present study were resistant to cefotaxime and 20\% were non- susceptible to meropenem in case of meningitis. These observations underline the importance of confirming susceptibility to beta-lactams in severe infections such as meningitis and septicemia.

When the prevalence of low-rPBP3 in Japanese respiratory isolates reached $17 \%$ in the mid 1990 s, group III isolates increased from zero to $29 \%$ in six years [13]. This was followed by a rapid increase in group III isolates in meningitis (predominantly Hib) from zero to $70 \%$ [15]. A recent report revealed a shift from low-level to high-level resistance in respiratory tract isolates in South Korea during the last decade, with an increase in the prevalence of group III isolates from $1 \%$ to $21 \%$ in five years $[16,22]$.

A similar development in other parts of the world would seriously compromise current empiric antibiotic therapy in severe infections. So far, single group III isolates have been reported from France [14] and Canada [3] whereas group III-like isolates are slightly more frequent $[9,14,18,20,21,24]$. Clusters of group III and group III-like high-level resistant isolates were recently observed in Norway (Skaare et al., manuscript in preparation).

The current epidemiologic situation in Europe and Canada, with a gradually increase in low-rPBP3 and sporadic reports of high-rPBP3 isolates, strongly resembles the situation in Japan and South Korea prior to the shifts in resistance genotypes. Continuous monitoring of susceptibility to cefotaxime and meropenem is necessary to ensure safe empiric treatment.

\section{Molecular epidemiology}

By comparing the study isolates with isolates from a comparable population collected in 2004 [11], we were able to study the clonal dynamics of PBP3-mediated resistance. The increasing prevalence of rPBP3 in Norway is due to expansion of a few clones. Four STs with characteristic ftsI alleles accounted for $61 \%$ of the rPBP3 isolates in the present study. Two of these strains were the main contributors to PBP3-mediated resistance in Norway three years earlier [11]. Interestingly, the replacement of ST14 by ST367 as the most prevalent rPBP3 strain did not cause a shift in PBP3 type nor phylogroup, as both STs carried PBP3 type A and belong to eBURST group 2.

We have previously suggested the existence of one or more widely disseminated rPBP3 clones [11]. This is supported by later reports of PBP3 type A and compatible

Table 4 Frequencies of beta-lactam resistance and clinical characteristics in study groups and in the original population ${ }^{a}$

\begin{tabular}{|c|c|c|c|c|c|c|c|c|c|c|c|}
\hline \multirow{3}{*}{ Groups of isolates $^{b}$} & \multirow{3}{*}{$n$} & \multicolumn{2}{|c|}{ rPBP3 $^{c}$} & \multicolumn{2}{|c|}{$B l a^{\mathrm{d}}$} & \multicolumn{6}{|c|}{ Proportions (\%) of isolates and patients } \\
\hline & & \multirow[t]{2}{*}{$n$} & \multirow[t]{2}{*}{$\%$} & \multirow[t]{2}{*}{$n$} & \multirow[t]{2}{*}{$\%$} & \multicolumn{3}{|c|}{ Anatomical sites } & \multicolumn{2}{|c|}{ Age groups } & \multirow[t]{2}{*}{ Hospitalized $^{\epsilon}$} \\
\hline & & & & & & Eye & Ear & Respiratory & $0-3$ & $\geq 50$ & \\
\hline Resistant group & 177 & 116 & 66 & 16 & 9 & 28 & 10 & 58 & 44 & 24 & 33 \\
\hline Susceptible group & 19 & 0 & 0 & 0 & 0 & 21 & 32 & 42 & 68 & 5 & 11 \\
\hline Remaining isolates & 599 & $0^{f}$ & $0^{f}$ & $60^{9}$ & $10^{\mathrm{g}}$ & 19 & 15 & 63 & 41 & 22 & 23 \\
\hline Original population & $795^{h}$ & 116 & 15 & 76 & 10 & 21 & 14 & 62 & 43 & 22 & 25 \\
\hline
\end{tabular}

${ }^{a}$ NORM 2007 surveillance population [33], consisting of consecutive routine isolates from patients with eye, ear and respiratory tract infections.

${ }^{b}$ See text and Figure 1 for definition of the study groups (Resistant group and Susceptible group).

'PBP3-mediated resistance (see Table 1).

dBeta-lactamase positive.

eProportions of patients hospitalized at the time of sampling.

${ }^{f}$ Assuming that all $\mathrm{rPBP} 3$ isolates were selected for the Resistant group.

${ }^{\mathrm{g}} \mathrm{As}$ reported by the primary laboratories.

${ }^{\mathrm{h}}$ Thirteen isolates were selected for the Resistant group but excluded for various reasons (see Figure 1). 
Table 5 Frequencies of beta-lactam resistance and clinical characteristics of study isolates according to STs

\begin{tabular}{|c|c|c|c|c|c|c|c|c|c|c|c|}
\hline \multirow{3}{*}{ STs } & \multirow{3}{*}{$n$} & \multicolumn{2}{|c|}{ rPBP3 $^{a}$} & \multicolumn{2}{|c|}{$B l a^{\mathbf{b}}$} & \multicolumn{6}{|c|}{ Proportions (\%) of isolates and patients ${ }^{c}$} \\
\hline & & \multirow[t]{2}{*}{$n$} & \multirow[t]{2}{*}{$\%$} & \multirow[t]{2}{*}{$n$} & \multirow[t]{2}{*}{$\%$} & \multicolumn{3}{|c|}{ Anatomical sites } & \multicolumn{2}{|c|}{ Age groups } & \multirow[t]{2}{*}{ Hospitalized $^{d}$} \\
\hline & & & & & & Eye & Ear & Respiratory & $0-3$ & $\geq 50$ & \\
\hline ST367 & 29 & 29 & 100 & 0 & 0 & 17 & 17 & 59 & 28 & 34 & 28 \\
\hline ST396 & 16 & 16 & 100 & 5 & 31 & $56^{e}$ & 6 & 38 & $81^{f}$ & 13 & 38 \\
\hline ST201 & 15 & 15 & 100 & 0 & 0 & $53^{e}$ & 0 & 47 & 47 & 27 & 47 \\
\hline ST159 & 12 & 1 & 8 & 0 & 0 & 8 & 8 & 75 & 33 & 42 & 50 \\
\hline ST14 & 11 & 11 & 100 & 1 & 9 & 18 & 0 & 73 & 64 & 9 & 55 \\
\hline ST12 & 8 & 7 & 88 & 0 & 0 & 50 & 13 & 38 & 38 & 13 & 25 \\
\hline ST395 & 8 & 0 & 0 & 0 & 0 & $63^{e}$ & 0 & 25 & 63 & 25 & 0 \\
\hline ST57 & 6 & 4 & 67 & 3 & 50 & 33 & 17 & 50 & 83 & 17 & 33 \\
\hline Other STs & 91 & 33 & 36 & 7 & 8 & 19 & 16 & 60 & 58 & 19 & 25 \\
\hline All STs & 196 & 116 & 59 & 16 & 8 & 27 & 12 & 56 & 46 & 22 & 31 \\
\hline
\end{tabular}

aPBP3-mediated resistance (see Table 1).

${ }^{\mathrm{b}}$ Beta-lactamase positive (all TEM-1).

'Proportions for each ST were compared with the proportions for other STs (e.g. ST396 versus non-ST396) using Fisher's exact test. Characteristics significantly

more prevalent in particular STs are indicated (bold).

dProportions of patients hospitalized at the time of sampling

$e_{p}<0.05$.

$f_{p}=0.004$.

substitution patterns (identical to PBP3 type A as far as comparison is possible) being common in Europe [4,18,23-25], Canada [3,12], Australia [20] and South Korea $[16,22]$, and by the present study.

PBP3 type A is frequently linked to ST14 and ST367 in the limited number of previous reports on the molecular epidemiology of rPBP3. Studies on invasive $H$. influenzae in Canada in the periods 2000-2006 [2,12,42] and 20082009 [3] revealed an increasing prevalence of rPBP3 in
NTHi, with PBP3 type A being common in both sampling periods [3,12]. ST14 and ST367, respectively, were the most common STs in NTHi from two different regions and sampling periods $[3,42]$. PBP3 type A was by far the most frequent substitution pattern in ST14 and also appeared in some ST367 isolates (R. Tsang, personal communication).

Furthermore, a study on invasive $H$. influenzae in Sweden [4] identified a cluster of seven NTHi isolates of

Table 6 Beta-lactam susceptibility according to PBP3 resistance genotypes

\begin{tabular}{|c|c|c|c|c|c|c|c|c|c|}
\hline \multirow[t]{2}{*}{ Study groups ${ }^{a}$} & \multirow{2}{*}{\multicolumn{2}{|c|}{ Resistance genotypes $^{\mathrm{b}}$}} & \multirow[t]{2}{*}{$n$} & \multicolumn{6}{|c|}{$\mathrm{MIC}_{50} / \mathrm{MIC}_{90}(\mathrm{mg} / \mathrm{L})$ and susceptibility categorization $(\%)^{\mathrm{c}}$} \\
\hline & & & & $\mathrm{AMP}^{\mathrm{C}}$ & $\mathrm{AMC}^{\mathrm{C}}$ & $\mathrm{PIP}^{c}$ & CXM & CTX & MEM \\
\hline \multirow[t]{10}{*}{ Resistant group } & High-rPBP3 & Group III & 1 & 8/- & $16 /-$ & $0.06 /-$ & $>16 /-$ & $0.25 /-$ & $1 /-$ \\
\hline & & & & $(0 / 100)$ & $(0 / 100)$ & & $(0 / 0 / 100)$ & $(0 / 100)$ & $(0 / 100 / 0)$ \\
\hline & & Group III-like & 2 & $2 / 4$ & $8 / 16$ & $0.06 / 0.12$ & $>16 />16$ & $0.06 / 0.12$ & $0.03 / 0.03$ \\
\hline & & & & $(0 / 100)$ & $(0 / 100)$ & & $(0 / 0 / 100)$ & $(100 / 0)$ & $(100 / 0 / 0)$ \\
\hline & Low-rPBP3 & Group II & 111 & $2 / 4$ & $4 / 8$ & $0.03 / 0.06$ & $8 / 8$ & $0.03 / 0.12$ & $0.12 / 0.5$ \\
\hline & & & & $(40 / 60)$ & $(45 / 55)$ & & $(33 / 11 / 56)$ & $(94 / 6)$ & $(80 / 20 / 0)$ \\
\hline & & Group I & 2 & $0.5 / 1$ & $0.25 / 1$ & $0.03 / 0.06$ & $0.5 / 16$ & $0.06 / 0.25$ & $0.016 / 0.06$ \\
\hline & & & & $(100 / 0)$ & $(100 / 0)$ & & $(50 / 0 / 50)$ & $(50 / 50)$ & $(100 / 0 / 0)$ \\
\hline & sPBP3 & & 60 & $0.25 / 0.5$ & $0.5 / 2$ & $0.004 / 0.03$ & $1 / 8$ & $0.008 / 0.06$ & $0.03 / 0.12$ \\
\hline & & & & $(98 / 2)$ & $(98 / 2)$ & & $(74 / 13 / 13)$ & $(98 / 2)$ & $(100 / 0 / 0)$ \\
\hline \multirow[t]{2}{*}{ Susceptible group } & sPBP3 & & 19 & $0.12 / 0.5$ & $0.5 / 2$ & $0.004 / 0.06$ & $0.5 / 8$ & $0.004 / 0.03$ & $0.03 / 0.12$ \\
\hline & & & & $(100 / 0)$ & $(95 / 5)$ & & $(79 / 11 / 11)$ & $(100 / 0)$ & $(100 / 0 / 0)$ \\
\hline
\end{tabular}

${ }^{\mathrm{a}}$ See Figure 1.

${ }^{\mathrm{b}} \mathrm{See}$ Table 1.

${ }^{\mathrm{c}} \mathrm{MICS}$ (microbroth dilution) and susceptibility categorization (S/R or S/I/R) according to EUCAST clinical breakpoints [37]. The following breakpoints were used $(\mathrm{S} \leq / \mathrm{R}>$ ): Ampicillin (AMP), 1/1; amoxicillin (AMC), 2/2; cefuroxime (CXM), 1/2; cefotaxime (CTX), 0.12/0.12; meropenem (MEM), 0.25/1. Clinical breakpoints for piperacillin and piperacillin-tazobactam are not set by EUCAST. Meningitis breakpoints were used for categorization of meropenem.

${ }^{d}$ For beta-lactamase positive isolates, ampicillin, amoxicillin and piperacillin MICs were determined in combination with sulbactam (4 mg/L), clavulanate ( 2 mg/L) and tazobactam (4 mg/L), respectively. 
ST14 and related STs (hereunder ST367), all carrying PBP3 type A and collected in the period 2008-2010 (F. Resman, personal communication). Finally, in two recently published Spanish studies, ST14 and/or ST367 isolates with substitution patterns compatible with PBP3 type A were reported in invasive disease (ST367, $n=2$ ) [24] and pneumonia (ST14, $n=2$; ST367, $n=1$ ) [25] in the period 2000-2009.

The ftsI alleles encoding type $\mathrm{A}$ in this and our previous study [11] had high genetic similarity and alleles in separate clusters rarely encoded identical PBP3 types. Thus, despite the lack of cross-study comparison of ftsI DNA sequences, the examples above indicate that clonal distribution is a more likely explanation for the occurrence of PBP3 type A and compatible patterns in separate studies from four continents [3,4,9,11,12,16,18,20,22-25] than independent development of this substitution pattern by convergence.

Importantly, an invasive high-level resistant $\mathrm{rPBP} 3$ isolate with the same combination of MLST allelic profile (ST155) and PBP3 substitution pattern as the two group III-like isolates in the present study was recently reported from Spain [24]. A single-locus variant (ST1118) with an identical substitution pattern was also reported.

These observations are notable and support the need of global surveillance initiatives. We here show that combining MLST and PBP3 typing provides a tool for cross-study identification of rPBP3 strains and clones. The previously suggested system for subgrouping of group II isolates [38] does not separate PBP3 types [11,16] and is unsuitable for this purpose.

Preferably, MLST should be combined with ftsI DNA sequencing. The ftsI gene is nearly $200 \mathrm{~kb}$ from its nearest MLST neighbor $(m d h)$ and distortion of the MLST results due to linkage is thus very unlikely. With recent technological development reducing both costs and analysis time of whole-genome sequencing, and smaller bench-top sequencers becoming readily available, MLST-ftsI typing will probably be possible to perform for surveillance purposes in the near future.

We are aware of a number of previous studies where MLST and ftsI sequencing was performed [3,4,12,23-25,43-45]. To our knowledge, four reports have linked MLST data and PBP3 substitution patterns: one presented the allelic profiles of 83 group III respiratory isolates from Japan [43]; another presented the substitution pattern of a single group II ST368 NTHi isolate causing meningitis in Italy [44]; and two most recent publications presented the substitution patterns and STs of 95 respiratory [25] and 18 invasive isolates [24] from Spain. However, the present study is to our knowledge the first to connect STs to ftsI alleles.
PFGE is highly discriminative and generally considered suited for assessment of relatedness between epidemiologically connected isolates, particularly in populations with high recombination rates such as NTHi $[39,46]$. In this study, PFGE clusters correlated well to MLST clonal complexes. Band patterns were stable over time and also traced phylogenetic relationship not detected by MLST and parsimony analysis. Combining MLST and PFGE for typing of NTHi may thus increase both sensitivity and resolution of clone detection.

\section{Development of resistance}

As discussed above, clonal expansion is important for the spread of rPBP3. However, the PBP3 type A-encoding, highly divergent ftsI allele lambda-2 was distributed among several unrelated STs. Similar observations are reported previously but only in studies using PFGE for strain characterization $[11,26]$. Exchange of complete alleles by HGT seems the most likely explanation, and has been demonstrated in vitro [26]. The mechanisms for HGT of ftsI sequences in $H$. influenzae are not completely resolved but involvement of classical transformation and homologous recombination has been suggested $[26,47]$.

Transformational competence varies extensively between H. influenzae strains [48]. This implies that the ability to acquire mutant ftsI alleles encoding rPBP3 will vary correspondingly, which may explain the differences in ST and phylogroup distribution between $\mathrm{rPBP} 3$ and sPBP3 isolates. It has been suggested that phylogroups are maintained by restriction barriers, preventing recombination between isolates of different heritage [32]. This is challenged by the distribution of lambda-2 to several phylogroups. A simple explanation may be that restriction barriers prevent recombination between some phylogroups and allow recombination between others.

Recent studies applying whole-genome sequencing have revealed that transformation in competent strains of $H$. influenzae is more extensive than previously recognized [49] and that transformational exchange may cause allelic variation involving complete genes between strains of identical STs [50]. However, transfer of complete ftsI alleles is probably less common than exchange of shorter sequences, causing mosaicism [26,28]. Preliminary multiple sequence alignment analysis of ftsI sequences in this study indicated intrageneic recombination (data not shown).

\section{PBP3-mediated resistance and virulence}

The association between $\mathrm{rPBP} 3$ and virulence is poorly described. One experimental study reported increased ability of a group III NTHi strain to invade bronchial epithelial cells, and the authors hypothesized that rPBP3 may enhance virulence by acting as an adhesion molecule [51]. 
A more recent retrospective epidemiological study concluded with no difference in pathogenicity between rPBP3 and SPBP3, but an association between rPBP3 and underlying respiratory disease was observed [17]. Molecular strain characterization was not performed in any of the two studies.

In the present study, regression analysis (without adjustment for ST) suggested that rPBP3 is associated with increased risk of eye infection and hospitalization. However, ST-specific analysis indicated that pathogenicity is correlated with STs rather than with resistance genotypes. For instance, ST395, ST396 and ST201 were significantly associated with eye infections but only the two latter STs were associated with PBP3-mediated resistance.

Irrespective of resistance genotypes, a number of STs in this study had clinical characteristics recognizable from previous reports: ST159 was predominantly isolated from the respiratory tract of hospitalized elderly patients, consistent with ST159 being adapted to infection in chronic obstructive pulmonary disease (COPD) [52]; and the high proportion of ST57 in children is consistent with a previously reported association with acute otitis media (AOM) [53]. Finally, the high hospitalization rate of patients with ST14-PBP3 type A corresponds well with the potential of this strain to cause pneumonia [25] and invasive disease $[3,4,42]$.

These observations are in accordance with a recent population study suggesting association between population structure and disease [53]. In conclusion, the association between $\mathrm{rPBP} 3$ and pathogenicity suggested by the regression analysis most likely reflects that some of the most frequently occurring rPBP3 strains in this study also possessed strain-associated virulence properties. Identification of virulence determinants is beyond the scope of this study. However, our observations underline that studies on the correlation between resistance genotypes and pathogenicity should include molecular strain characterization. Accordingly, the previously reported association between PBP3-mediated resistance and clinical characteristics $[17,51]$ may be spurious.

\section{Conclusions}

The prevalence of rPBP3 in $H$. influenzae is increasing worldwide, and high-level resistant strains are emerging in new geographic regions.

In this study of eye, ear and respiratory isolates in Norway, the rPBP3 prevalence was $15 \%$, with four strains accounting for $61 \%$ of the resistant isolates. Group II lowrPBP3 isolates predominated, and significant proportions of isolates were non-susceptible to cefotaxime and meropenem. Group III high-rPBP3 was identified for the first time in Northern Europe.
The results support a role of horizontal gene transfer in the emergence of rPBP3 and indicate phylogeny restricted transformation. Comparative analysis with data from previous studies indicates wide dissemination of clonally related rPBP3 strains. Notably, two strains highly prevalent in Norway (ST14 and ST367 with PBP3 type A) are common in invasive disease in Europe and Canada.

Continuous monitoring of beta-lactam susceptibility is necessary to ensure safe empiric therapy in severe disease and to detect a future shift from low-level to high-level resistance. The need of a global system for molecular surveillance of rPBP3 strains is underlined. The novel approach of combining MLST and ftsI/PBP3 typing is a powerful tool for this purpose.

\section{Abbreviations \\ NTHi: Nontypeable Haemophilus influenzae; Hib: Haemophilus influenzae serotype b; Hif: Haemophilus influenzae serotype f; PBP3: Penicillin-binding protein 3; BLNAR: Beta-lactamase negative ampicillin resistant; BLPACR: Beta-lactamase positive amoxicillin-clavulanate resistant; rPBP3: PBP3- mediated resistance present ('resistant' PBP3); sPBP3: PBP3-mediated resistance absent ('susceptible' PBP3); MLST: Multilocus sequence typing; ST: Sequence type; CC: Clonal complex; Phylogroup: Phylogenetic group; PFGE: Pulsed-field gel electrophoresis; HGT: Horizontal gene transfer; UPGMA: Unweighted pair group method with arithmetic mean; EUCAST: European Committee on Antimicrobial Susceptibility Testing; MIC: Minimal inhibitory concentration; COPD: Chronic obstructive pulmonary disease; AOM: Acute otitis media; NORM: Norwegian Surveillance Programme for Antimicrobial Resistance; R-group: Resistant group; S-group: Susceptible group.}

\section{Competing interests}

The authors declare that they have no competing interests.

\section{Authors' contributions}

DS conceived and coordinated the study, performed susceptibility testing analysed and interpreted data and wrote the first draft; BEK, YT, AJ, LS and AS contributed to study design; ILA designed and undertook molecular analyses (except MLST); LS analysed the PFGE data, DAC and MS were responsible for acquisition of MLST data and AJ advised on bioinformatics. All authors participated in interpretation of results, critically revised the draft for intellectual content and approved the final article.

\section{Acknowledgements}

The work was supported by grants from Vestfold Hospital Trust, University of Tromsø, the Scandinavian Society for Chemotherapy (SSAC), and the Norwegian Surveillance Programme for Antimicrobial Resistance (NORM). We thank the staff at the laboratories contributing with isolates; NORM for access to the surveillance database; Raymond S. W. Tsang and Fredrik Resman for sharing data; and the following for excellent technical assistance: Astrid Lia, Anja Hannisdal and Wenche Petterson (susceptibility testing, handling of isolates etc.); Anne Gry Allum (PFGE) and Martha Langedok Bjørnstad (MLST).

\section{Author details}

'Department of Microbiology, Vestfold Hospital Trust, Tønsberg, Norway. ${ }^{2}$ University of Tromsø, Tromsø, Norway. ${ }^{3}$ Department of Bacteriology and Immunology, Norwegian Institute of Public Health, Oslo, Norway. ${ }^{4}$ Faculty of Medicine, University of Oslo, Oslo, Norway. ${ }^{5}$ Department of Environmental and Health Sciences, Telemark University College, Bø, Norway. ${ }^{6}$ Telemark Hospital, Skien, Norway.

Received: 10 January 2014 Accepted: 14 May 2014 Published: 20 May 2014 


\section{References}

1. Jordens JZ, Slack MPE: Haemophilus influenzae: Then and now. Eur J Clin Microbiol Infect Dis 1995, 14:935-948.

2. Sill ML, Tsang RSW: Antibiotic susceptibility of invasive Haemophilus influenzae strains in Canada. Antimicrob Agents Chemother 2008, 52:1551-1552

3. Shuel M, Hoang L, Law DKS, Tsang R: Invasive Haemophilus influenzae in British Columbia: non-Hib and non-typeable strains causing disease in children and adults. Int J Infect Dis 2011, 15:e167-e173.

4. Resman F, Ristovski M, Forsgren A, Kaijser B, Kronvall G, Medstrand P, Melander E, Odenholt I, Riesbeck K: Increase of beta-lactam-resistant invasive Haemophilus influenzae in Sweden, 1997 to 2010. Antimicrob Agents Chemother 2012, 56:4408-4415.

5. Murphy T: Current and future prospects for a vaccine for nontypeable Haemophilus influenzae. Curr Infect Dis Rep 2009, 11:177-182.

6. Tristram S, Jacobs MR, Appelbaum PC: Antimicrobial resistance in Haemophilus influenzae. Clin Microbiol Rev 2007, 20:368-389.

7. Ubukata K, Shibasaki Y, Yamamoto K, Chiba N, Hasegawa K, Takeuchi Y, Sunakawa K, Inoue M, Konno M: Association of amino acid substitutions in penicillin-binding protein 3 with beta-lactam resistance in beta-lactamase-negative ampicillin-resistant Haemophilus influenzae. Antimicrob Agents Chemother 2001, 45:1693-1699.

8. Hasegawa K, Chiba N, Kobayashi R, Murayama SY, Iwata S, Sunakawa K, Ubukata K: Rapidly increasing prevalence of beta-lactamase-nonproducing, ampicillin-resistant Haemophilus influenzae type $b$ in patients with meningitis. Antimicrob Agents Chemother 2004, 48:1509-1514.

9. Garcia-Cobos S, Campos J, Lazaro E, Roman F, Cercenado E, Garcia-Rey C, Perez-Vazquez M, Oteo J, de AF: Ampicillin-resistant non-beta-lactamaseproducing Haemophilus influenzae in Spain: recent emergence of clonal isolates with increased resistance to cefotaxime and cefixime. Antimicrob Agents Chemother 2007, 51:2564-2573.

10. Hotomi M, Fujihara K, Billal DS, Suzuki K, Nishimura T, Baba S, Yamanaka N: Genetic characteristics and clonal dissemination of beta-lactamase non-producing ampicillin-resistant (BLNAR) Haemophilus influenzae isolated from the upper respiratory tract in Japan. Antimicrob Agents Chemother 2007, 51:3969-3976.

11. Skaare D, Allum AG, Anthonisen IL, Jenkins A, Lia A, Strand L, Tveten Y, Kristiansen BE: Mutant fts/ genes in the emergence of penicillin-binding protein-mediated beta-lactam resistance in Haemophilus influenzae in Norway. Clin Microbiol Infect 2010, 16:1117-1124.

12. Shuel ML, Tsang RSW: Canadian beta-lactamase negative Haemophilus influenzae isolates showing decreased susceptibility toward ampicillin have significant penicillin binding protein 3 mutations. Diagn Microbiol Infect Dis 2009, 63:379-383.

13. Ubukata K: Problems associated with high prevalence of multidrug-resistant bacteria in patients with community-acquired infections. I Infect Chemother 2003, 9:285-291.

14. Dabernat H, Delmas C: Epidemiology and evolution of antibiotic resistance of Haemophilus influenzae in children 5 years of age or less in France, 2001-2008: a retrospective database analysis. Eur J Clin Microbiol Infect Dis 2012, 31:2745-2753.

15. Ubukata K, Chiba N, Morozumi M, Iwata S, Sunakawa K: Longitudinal surveillance of Haemophilus influenzae isolates from pediatric patients with meningitis throughout Japan, 2000-2011. J Infect Chemother 2013, 19:34-41.

16. Park C, Kim KH, Shin NY, Byun JH, Kwon EY, Lee JW, Kwon HJ, Choi EY, Lee DG, Sohn WY, Kang JH: Genetic diversity of the ftsl gene in beta-lactamase-nonproducing ampicillin-resistant and beta-lactamaseproducing amoxicillin-/clavulanic acid-resistant nasopharyngeal Haemophilus influenzae strains isolated from children in South Korea. Microb Drug Resist 2013, 19:224-230.

17. Hagiwara E, Baba T, Shinohara T, Nishihira R, Komatsu S, Ogura T: Antimicrobial resistance genotype trend and its association with host clinical characteristics in respiratory isolates of Haemophilus influenzae. Chemotherapy 2012, 58:352-357.

18. Barbosa AR, Giufre M, Cerquetti M, Bajanca-Lavado MP: Polymorphism in $\mathrm{ftsl}$ gene and beta-lactam susceptibility in Portuguese Haemophilus influenzae strains: clonal dissemination of beta-lactamase-positive isolates with decreased susceptibility to amoxicillin/clavulanic acid. J Antimicrob Chemother 2011, 66:788-796.
19. Kaczmarek FS, Gootz TD, Dib-Hajj F, Shang W, Hallowell S, Cronan M: Genetic and molecular characterization of beta-lactamase-negative ampicillin-resistant Haemophilus influenzae with unusually high resistance to ampicillin. Antimicrob Agents Chemother 2004, 48:1630-1639.

20. Witherden EA, Montgomery J, Henderson B, Tristram SG: Prevalence and genotypic characteristics of beta-lactamase-negative ampicillin-resistant Haemophilus influenzae in Australia. J Antimicrob Chemother 2011, 66:1013-1015

21. Sevillano D, Giménez MJ, Cercenado E, Cafini F, Gené A, Alou L, Marco F, Martinez-Martinez L, Coronel P, Aguilar L: Genotypic versus phenotypic characterization, with respect to beta-lactam susceptibility, of Haemophilus influenzae isolates exhibiting decreased susceptibility to betalactam resistance markers. Antimicrob Agents Chemother 2009, 53:267-270.

22. Bae S, Lee J, Lee J, Kim E, Lee S, Yu J, Kang Y: Antimicrobial resistance in Haemophilus influenzae respiratory tract isolates in Korea: results of a nationwide acute respiratory infections surveillance. Antimicrob Agents Chemother 2010, 54:65-71.

23. Bajanca-Lavado MP, Simoes AS, Betencourt CR, Sa-Leao R: Characteristics of Haemophilus influenzae invasive isolates from Portugal following routine childhood vaccination against $H$. influenzae serotype b (2002-2010). Eur J Clin Microbiol Infect Dis 2014, 33:603-610.

24. Garcia-Cobos S, Arroyo M, Perez-Vazquez M, Aracil B, Lara N, Oteo J, Cercenado E, Campos J: Isolates of beta-lactamase-negative ampicillinresistant Haemophilus influenzae causing invasive infections in Spain remain susceptible to cefotaxime and imipenem. J Antimicrob Chemother 2014, 69:111-116.

25. Puig C, Calatayud L, Marti S, Tubau F, Garcia-Vidal C, Carratala J, Linares J, Ardanuy C: Molecular epidemiology of nontypeable Haemophilus influenzae causing community-acquired pneumonia in adults. PLOS One 2013, 8:e82515.

26. Takahata S, Ida T, Senju N, Sanbongi Y, Miyata A, Maebashi K, Hoshiko S: Horizontal gene transfer of $f t s l$, the gene encoding penicillin-binding protein 3, in Haemophilus influenzae. Antimicrob Agents Chemother 2007, 51:1589-1595.

27. Sanbongi Y, Suzuki T, Osaki Y, Senju N, Ida T, Ubukata K: Molecular evolution of beta-lactam-resistant Haemophilus influenzae: 9-year surveillance of penicillin-binding protein 3 mutations in isolates from Japan. Antimicrob Agents Chemother 2006, 50:2487-2492.

28. Witherden EA, Bajanca-Lavado MP, Tristram SG, Nunes A: Role of inter-species recombination of the ftsl gene in the dissemination of altered penicillinbinding-protein-3-mediated resistance in Haemophilus influenzae and Haemophilus haemolyticus. J Antimicrob Chemother 2014, 69:1501-1509.

29. Harrison $O B$, Brueggemann $A B$, Caugant $D A$, van der Ende A, Frosch $M$, Gray S, Heuberger S, Krizova P, Olcen P, Slack M, Taha MK, Maiden MCJ: Molecular typing methods for outbreak detection and surveillance of invasive disease caused by Neisseria meningitidis, Haemophilus influenzae and Streptococcus pneumoniae, a review. Microbiology 2011, 157:2181-2195.

30. Meats E, Feil EJ, Stringer S, Cody AJ, Goldstein R, Kroll JS, Popovic T, Spratt BG: Characterization of encapsulated and noncapsulated Haemophilus influenzae and determination of phylogenetic relationships by multilocus sequence typing. J Clin Microbiol 2003, 41:1623-1636

31. Feil EI, Li BC, Aanensen DM, Hanage WP, Spratt BG: eBURST: inferring patterns of evolutionary descent among clusters of related bacterial genotypes from multilocus sequence typing data. J Bacteriol 2004, 186:1518-1530.

32. Erwin AL, Sandstedt SA, Bonthuis PJ, Geelhood JL, Nelson KL, Unrath WCT, Diggle MA, Theodore MJ, Pleatman CR, Mothershed EA, Sacchi CT, Mayer LW, Gilsdorf JR, Smith AL: Analysis of genetic relatedness of Haemophilus influenzae isolates by multilocus sequence typing. J Bacteriol 2008, 190:1473-1483.

33. NORM/NORM-VET 2007: Usage of Antimicrobial Agents and Occurrence of Antimicrobial Resistance in Norway. Tromsø/Oslo, Norway. 2008. http://www.unn.no/getfile.php/UNN\%20INTER/Fagfolk/www. antibiotikaresistens.no/NORM\%202008/NORM\%20NORM-VET\%202007.pdf.

34. Stralin K, Backman A, Holmberg H, Fredlund H, Olcen P: Design of a multiplex PCR for Streptococcus pneumoniae, Haemophilus influenzae, Mycoplasma pneumoniae and Chlamydophila pneumoniae to be used on sputum samples. APMIS 2005, 113:99-111.

35. Falla TJ, Crook DW, Brophy LN, Maskell D, Kroll JS, Moxon ER: PCR for capsular typing of Haemophilus influenzae. J Clin Microbiol 1994, 32:2382-2386. 
36. Clinical and Laboratory Standards Institute: Performance standards for antimicrobial susceptibility testing, twenty-third informational supplement. CLSI document M100-S23. 2013.

37. The European Committee on Antimicrobial Susceptibility Testing (EUCAST): Breakpoint tables for interpretation of MICs and zone diameters. Version 4.0, 2014. 2014. http://www.eucast.org.

38. Dabernat H, Delmas C, Seguy M, Pelissier R, Faucon G, Bennamani S, Pasquier C: Diversity of beta-lactam resistance-conferring amino acid substitutions in penicillin-binding protein 3 of Haemophilus influenzae. Antimicrob Agents Chemother 2002, 46:2208-2218.

39. Tenover FC, Arbeit RD, Goering RV, Mickelsen PA, Murray BE, Persing DH, Swaminathan B: Interpreting chromosomal DNA restriction patterns produced by pulsed-field gel electrophoresis: criteria for bacterial strain typing. J Clin Microbiol 1995, 33:2233-2239.

40. NORM/NORM-VET 2011: Usage of Antimicrobial Agents and Occurrence of Antimicrobial Resistance in Norway. Tromsø/Oslo, Norway. 2012. http://www.unn.no/getfile.php/UNN\%20INTER/Fagfolk/www. antibiotikaresistens.no/NORM\%202012/NORM\%20NORM-VET\%202011.pdf.

41. Norwegian Institute of Public Health: Årsrapport 2012 for sykdomsprogrammet Invasive sykdommer. Oslo, Norway. 2013. http://www.fhi.no/dokumenter/045999cadf.pdf.

42. Sill ML, Law DKS, Zhou J, Skinner S, Wylie J, Tsang RSW: Population genetics and antibiotic susceptibility of invasive Haemophilus influenzae in Manitoba, Canada, from 2000 to 2006. FEMS Immun \& Med Microbiol 2007, 51:270-276.

43. Sunakawa K, Farrell DJ: Mechanisms, molecular and sero-epidemiology of antimicrobial resistance in bacterial respiratory pathogens isolated from Japanese children. Ann Clin Microbiol Antimicrob 2007, 6:7.

44. Cardines R, Giufre M, Mastrantonio P, Gli Atti ML, Cerquetti M: Nontypeable Haemophilus influenzae meningitis in children: phenotypic and genotypic characterization of isolates. Pediatr Infect Dis J 2007, 26:577-582.

45. Otsuka T, Komiyama K, Yoshida K, Ishikawa Y, Zaraket H, Fujii K, Okazaki M: Genotyping of Haemophilus influenzae type $b$ in pre-vaccination era. J Infect Chemother 2012, 18:213-218.

46. Thomas J, Pettigrew M: Multilocus sequence typing and pulsed field gel electrophoresis of otitis media causing pathogens. In Auditory and Vestibular Research. 493rd edition. Edited by Sokolowski B. New York: Humana Press; 2009:179-190.

47. Osaki Y, Sanbongi Y, Ishikawa M, Kataoka H, Suzuki T, Maeda K, Ida T: Genetic approach to study the relationship between penicillin-binding protein 3 mutations and Haemophilus influenzae beta-lactam resistance by using site-directed mutagenesis and gene recombinants. Antimicrob Agents Chemother 2005, 49:2834-2839.

48. Maughan $\mathrm{H}$, Redfield RJ: Extensive variation in natural competence in Haemophilus influenzae. Evolution 2009, 63:1852-1866.

49. Mell JC, Shumilina S, Hall IM, Redfield RJ: Transformation of natural genetic variation into Haemophilus influenzae genomes. PLoS Pathog 2011, 7:e1002151.

50. Power P, Bentley S, Parkhill J, Moxon E, Hood D: Investigations into genome diversity of Haemophilus influenzae using whole genome sequencing of clinical isolates and laboratory transformants. BMC Microbiol 2012, 12:273.

51. Okabe T, Yamazaki Y, Shiotani M, Suzuki T, Shiohara M, Kasuga E, Notake S, Yanagisawa H: An amino acid substitution in PBP-3 in Haemophilus influenzae associate with the invasion to bronchial epithelial cells. Microbiol Res 2010, 165:11-20.

52. Murphy TF, Lesse AJ, Kirkham C, Zhong H, Sethi S, Munson RS: A clonal group of nontypeable Haemophilus influenzae with two $\lg A$ proteases is adapted to infection in chronic obstructive pulmonary disease. PLoS One 2011, 6:e25923.

53. LaCross NC, Marrs CF, Gilsdorf JR: Population structure in nontypeable Haemophilus influenzae. Infect Genet Evol 2013, 14:125-136.

\section{doi:10.1186/1471-2180-14-131}

Cite this article as: Skaare et al:: Multilocus sequence typing and ftsl sequencing: a powerful tool for surveillance of penicillin-binding protein 3-mediated beta-lactam resistance in nontypeable Haemophilus influenzae. BMC Microbiology 2014 14:131.

\section{Submit your next manuscript to BioMed Central and take full advantage of:}

- Convenient online submission

- Thorough peer review

- No space constraints or color figure charges

- Immediate publication on acceptance

- Inclusion in PubMed, CAS, Scopus and Google Scholar

- Research which is freely available for redistribution 